\title{
Mirror Neurons, Prediction and Hemispheric Coordination: The Prioritizing of Intersubjectivity Over 'Intrasubjectivity'
}

\author{
Richard Shillcock ${ }^{1} \cdot$ James Thomas ${ }^{1} \cdot$ Rachael Bailes $^{1}$
}

Received: 30 April 2018 / Accepted: 18 November 2018 / Published online: 24 November 2018

(C) The Author(s) 2018

\begin{abstract}
We observe that approaches to intersubjectivity, involving mirror neurons and involving emulation and prediction, have eclipsed discussion of those same mechanisms for achieving coordination between the two hemispheres of the human brain. We explore some of the implications of the suggestion that the mutual modelling of the two situated hemispheres (each hemisphere 'second guessing' the other) is a productive place to start in understanding the phylogenetic and ontogenetic development of cognition and of intersubjectivity.
\end{abstract}

Keywords Hemisphericity $\cdot$ Intersubjectivity $\cdot$ Mirror neurons $\cdot$ Predictive processing $\cdot$ Emulation

Research on intersubjectivity has received influential contributions from two sources over recent decades. First, from research on the mirror neuron system (MNS) (e.g. Fadiga et al. 1995; Gallese et al. 1996). Second, from the resurgence of interest in emulation and prediction (e.g. Clark 2013; Friston 2005; Grush 2004; Rao and Ballard 1999; Lee and Mumford 2003). In both cases, specific mechanisms have been proposed as foundational for much of what is understood as intersubjectivity. In this paper we highlight the fact that this concentration on intersubjectivity has been at the expense of what we can call intrasubjectivity-the necessary coordination of the activity of the two hemispheres of the human brain. We claim that the relevant mechanisms of the MNS and of emulation and prediction (or 'modelling,'

Richard Shillcock

rcs@inf.ed.ac.uk

https://sites.google.com/site/rcspplsinf/home

James Thomas

notrevolution@gmail.com

Rachael Bailes

Rachael.Bailes@newcastle.ac.uk

1 School of Philosophy Psychology and Language Sciences, The University of Edinburgh, 7

George Square, Edinburgh EH8 9JZ, Scotland, UK 
more broadly) can be productively seen as mechanisms of hemispheric coordination that are in many ways prior to and foundational for intersubjectivity and for wider cognition. This view of the development of human cognition, both phylogenetic and ontogenetic, sees each hemisphere 'second-guessing' the other in a largely shared external and internal (embodied) context.

\section{The Hemispheric Divide}

Initially, we establish why it is important to consider hemisphericity in connection with the uniqueness of human cognition. First, there is the increasing independence of the two hemispheres in non-primate and primate evolution (Aboitiz and Montiel 2003; Hopkins and Rilling 2000; Ringo et al. 1994; Rilling and Insel 1999), as defined by the size relation of the corpus callosum and the neocortex. Larger brains do not have proportionately more callosal connections. ${ }^{1}$

Second, asymmetry. A vertical axis of symmetry began at least in the Burgess Shale. Asymmetry of structure/function about the vertical midline is widespread (e.g. Denenberg 1981; MacNeilage et al. 2009), from nematode worms (Hobert et al. 2002), through to great apes (Quaresmini et al. 2014). However, this asymmetry is greatest in human lateral cerebral specialization: "Nowhere in the animal kingdom is there such rampant specialization of function" (Gazzaniga 2000, p. 1294).

Third, there is the very plastic interdependence of the two hemispheres. Callosal agenesis and related disorders have very variable effects on cognition, sometimes having no apparent effects on IQ and at other times profoundly impairing it (Chiarello 1980; Sauerwein et al. 1994). Actual hemispherectomy sometimes seems not to have such drastic consequences as might be imagined (e.g. Pulsifer et al. 2004). Adult callosotomy produces the well-known disconnection syndrome, in which the individual is left paradoxically intact, requiring ingenious experiments to reveal the cognitive implications of the severing of the corpus callosum (e.g., Gazzaniga 2005; Gazzaniga et al. 1962, 1963, 1965; Gazzaniga and Sperry 1967; Sperry 1964). Even here, though, there seems to be individual variation in its effects on, for example, resting state activity (see Johnson et al. 2008; Uddin et al. 2008). This very variability of hemispheric interaction alerts us to its potential.

Schechter (2015) rehearses the route by which psychologists (including 'folk psychologists') readily accepted the duality of the brain and its functions following the influential split-brain studies (e.g. Sperry 1965; Bogen 1969; Gazzaniga 1970), whereas philosophers have been more sceptical that the mind can be similarly divided (e.g. Hurley 2002; Marks 1981; Tye 2003). Schechter makes the case for the callosal syndrome observed in split-brain individuals involving the hemispheres operating independently and being mediated by "effection and transduction"

\footnotetext{
1 Although humans have easily the greatest hemispheric independence compared with evolutionarily related species, Rilling and Insel note even greater hemispheric independence in delphinid cetaceans, but without relatively sophisticated cognition. Other factors are necessary-possibly having an ever-increasing spiral of problems to solve in the world and the artefacts with which to solve them.
} 
(behavior and sensation), and hence qualifying as "two minds." In other work, she addresses the unity of consciousness and argues that "a subject might possess a single stream of consciousness that was still disunified, or a significantly unified consciousness that was nonetheless comprised of multiple streams of consciousness" (Schechter 2013, p. 214). To ground out some of these intuitions anatomically, Schechter takes as candidate cognizing entities " $R$ and L, where $R$ is the entire subject minus the left hemisphere and $\mathrm{L}$ is the entire split-brain subject minus the right hemisphere" (p. 513), citing Davis (1997).

In short, hemisphericity in humans is a point of maximal departure from even the highest other primates. The two hemispheres exhibit surprising levels of independence and plasticity. Their coordination is a necessity and is replete with possibilities, both philosophical and psychological.

\section{The Range of Inter-hemispheric Connectivity}

The apparent unity of consciousness encourages cognitive scientists to view the normal brain as a neuronally mediated whole, with callosal (and other) connectivity underlying the apparent equipotentiality of metacognition and consciousness. Theorists taking an abstract computational perspective have conventionally thought of disembodied information washing backwards and forwards between the hemispheres, also making the brain effectively a single domain (e.g. Reggia et al. 1998).

The sheer range of anatomical connectivity between the hemispheres supports the view of the brain as a single domain. Although the corpus callosum is the most conspicuous commissure, it is not alone (cf. Trevarthen 1990) and others may compensate for it (e.g. Loeser and Alvord 1968; although see Kingstone and Gazzaniga 1995). Recent years have seen an appreciation of the role of the cerebellum in cognition (e.g. Middleton and Strick 2000); the cerebellum has left-right structure and predominantly unilateral connectivity, but there is also ipsilateral connectivity (Glickstein 2009; Savaki et al. 1996). There is rich subcortical connectivity between the two sides of the nervous system, also interacting vertically with the respective cerebral hemispheres (e.g. Pashler et al. 1994). The totality of routes by which the two hemispheres coordinate themselves inescapably involves the outside world: callosal, cerebral and subcortical connections directly or indirectly involve all sorts of sensory data; changing eye-fixation to inspect different parts of the world moves the visual contents of one hemifield/hemisphere to the other hemisphere; autonomic nervous system activity affects the bodily state available to both hemispheres (Gazzaniga 2002, p. 210); kinaesthetic and proprioceptive functioning embody information about whole-body posture and activity; self-directed speech can organize activity; the individual's physical interaction with the world of people and artefacts can mediate hemispheric interaction, as in the split-brain 'cross-cueing' examples (the right hemisphere $[\mathrm{RH}]$ instructs the left hand to stroke an unseen comb, and the left hemisphere [LH] verbally identifies the sound); we see an enlargement of the anterior corpus callosum in musicians trained from their early years, suggesting that particular activity can forge closer coordination between the hemispheres (Schlaug et al. 1995). 
These various communication routes between the two sides of the nervous system still do not necessitate regarding the brain as a unified whole, however. For instance, Whitteridge (1965) reports that neurons earlier in the visual pathway need to have a receptive field that contains a representation of the midline between the two hemifields for them to have a callosal projection; in this way the corpus callosum draws together the two separate hemifield curtains of the visual field into a single, unified visual experience. The farther we move up the visual pathways from the sensory input to the higher visual areas, the more we find neurons with receptive fields that respond to both left and right hemifields, as opposed to solely the contralateral hemifield, although we typically still see a stronger contralateral response (Berlucchi and Antonini 1990). Fundamentally, though, the cleanly-divided primary visual cortex remains the most authoritative reflection of the visual world; it is the target of substantial 'top-down' feedback within the visual system (Felleman and Van Essen 1991) and is intimately involved with the generation of conscious visual experience (Tong 2003). The callosal disconnection syndrome remains the most striking argument for the potential independence of the two hemispheres.

Overall, we see rich connectivity between the two hemispheres, co-located in the same internal environment of hormones, bodily postures and movements, and in the same external environment of sights, sounds, touches and smells. In these circumstances the observed degree of hemispheric differentiation is all the more striking.

Note that the individual has a degree of active control over hemispheric coordination, mediated by artefacts and social interaction. From the earliest work on callosal disconnection, researchers have seen the individual's own bodily activity as crucial to cerebral unity (e.g. Sperry et al. 1969, p. 286), but we should also consider its potential role in keeping distinct the activities of the two hemispheres; in this respect, individual- (as opposed to population-) level handedness is a very human characteristic (Amunts et al. 2000; Cashmore et al. 2008; Cavanagh et al. 2015).

We have seen that the hemispheric divide, together with the structural and function asymmetry it allows, is a salient material feature of the human brain. It necessitates coordination. We have also seen rich hemispheric connectivity, but without any recognizable, principled mechanism (with the possible exception of head-turning to 'transfer' visual information). Thus far, hemisphericity looks like a problem. In what sense does it represent an opportunity?

\section{Hemispheric Homology and Bandwidth}

Before we can approach the mutual modelling that we will claim is the opportunity present in hemisphericity, we need to review two more respects in which the hemispheric divide is special. Why is the division between two individuals, or between any two distinct brain regions, not at least as important as the division between the hemispheres? Why is the latter so important?

The key arguments concern homology and bandwidth. Homology refers to the two hemispheres being structural mirror-images. Functional hemispheric differences exist in the neonate (Witelson and Pallie 1973) and also emerge over development (e.g. Mevorach et al. 2005), producing different parameterizations of the 
hemispheres (e.g. Beeman et al. 1994), but, even given the existence of an eventual hemispheric division of labour potentially presided over by Levy and Trevarthen's (1976) "metacontrol", each hemisphere is fundamentally capable of doing more or less anything its partner can do, albeit sometimes not quite as well (cf. Beeman and Chiarello 1998; Hertz-Pannier et al. 2002).

Even given the plasticity of the neocortex (Kolb and Whishaw 1989), homology does not occur anywhere near as fully in, for instance, the relationship between the frontal cortex and the inferior temporal cortex, or in any other subsidiary relationship within the nervous system. The homology between the hemispheres is maximal, and of special significance.

Surely the homology argument applies between subjects? As with the hemispheres, each individual is fundamentally capable of doing more or less anything another individual can do. Surely the range of the homology between individual subjects is greater than between hemispheres?

There are two points to make. First, adult caregivers can do a lot, infants can do very little, so any activity-mediated homology is limited. Neither infant hemisphere can control many actions, but the given homology between them is nevertheless considerable. Clearly the homology between adult caregiver and infant increases during development, and the relationship between two adult subjects eventually encompasses the whole culture. Understanding that relationship is an ultimate goal of cognitive scientists, but our central argument here is that this understanding is productively approached through considering hemispheric coordination.

Second, even a face-to-face homology between two independent subjects, is still appropriately thought of in hemispheric terms. When Subject A centrally fixates Subject B's face, then the contralateral projection of the vertically divided visual field to A's hemispheres means that A's right and left hemispheres are 'directly speaking to' B's left and right hemispheres, respectively, given that B's face (and body) are primarily contralaterally controlled by B's cortical hemispheres. This relationship between the hemispheres of different individuals recapitulates the prior, much more intimate relationship between A's own RH and LH. (We might consider the 'mirror twins' Greta and Freda Chaplin-anecdotally reported by Brook and Raymont 2017 — as the atypical extreme of the mutual entrainment of two pairs of hemispheres.)

In addition to homology, understanding hemispheric coordination requires us to consider the bandwidth of the interactions. Eliasmith (2008, p. 150), Chalmers (2008, p. xii) and Clark (2009, pp. 983-984) all refer to "bandwidth"-the speed and richness of interaction-as an argument for restricting to the brain that which is constitutive of consciousness. An analogous bandwidth argument applies to hemispheric interaction. Interhemispheric interaction is necessarily richer than most of the interactions between domains smaller than the hemispheres. Interaction between two subjects is necessarily sparse and relatively slow; however, it is 'high-level' - as in language. But even this case does not necessarily beat hemispheric interaction for richness, as self-talk can still organize cognition.

Only very general principles of the how? and the where? of hemispheric interaction have emerged (cf. Davis and Cabeza 2015), and then only slowly, from what was long seen as a broadly homotopic and inhibitory relationship between 
the hemispheres (cf. Chiarello and Maxfield 1996; Cook 1986; Marcano-Reik and Blumberg 2008). Why is there not more transparent functional motivation in the connectivity of the corpus callosum? How much detailed hardwiring should we expect?

A growing number of studies involving brain-to-brain interfaces show that very broad-brush ways of picking up information from a 'sending' brain and of influencing a 'receiving' brain can be effective in enabling purposeful communications between two rat brains (Pais-Vieira et al. 2013), between a human brain and a rat brain (Yoo et al. 2013), and between two human brains (Rao et al. 2014; see, also, Min et al. 2010). Given the number of callosal connections and the range of noncallosal physiological and anatomical routes by which one hemisphere may influence another, there is little doubt that there is the wherewithal for developing novel, effective hemisphere-to-hemisphere communication without elaborate pre-wiring. Indeed, such pre-wiring may make the relationship too 'brittle' to succeed phylogenetically and ontogenetically.

In summary, the interface between the two hemispheres is salient because this interface has maximal bandwidth and maximal opportunity for the interaction of processing differences. Indeed, two half-spheres with homologous structure and connectivity has a good claim to be the optimal compromise between difference and speed of interaction. ${ }^{2}$ Only minimal pre-existing structure seems to be required as a foundation for effective hemispheric communication.

\section{Algorithmic Analogies}

The fields of computational cognitive modelling, statistical physics and machine learning contain implementations of some of the principles considered above. There is a history of neural net simulations with a 'hemispheric split' (Anninos and Cook 1988; Monaghan et al. 2004; Reggia et al. 2013; Shillcock and Monaghan 2001; Monaghan and Pollmann 2003; Shillcock et al. 2018; Shkuro et al. 2000), in which typically the two halves of the model are encapsulated, except for a set of 'callosal' connections between the sets of hidden units. One half of the model 'second guesses' the other half.

As another example, the "complexity matching" developed by West et al. (Allegrini et al. 2006; West et al. 2008) in the statistical physics of complex networks, demonstrates that there is maximal information exchange between coupled complex systems each of which generates similar power-law relationships between variables in its own domain of processing. Cognitive scientists have applied

\footnotetext{
${ }^{2}$ Consider a population of red-blue dipoles, in which difference is accentuated by the relative proximity of all the red poles and all the blue poles, respectively. The optimum structure is a sphere of all the red poles and a sphere of all the blue poles. Speed is inversely proportional to the distance between the two poles of each dipole. The optimum structure begins as the two spheres with a one-to-one 'straight line' mapping between the poles of each dipole, which is then compressed into two half-spheres to shorten the distance between the poles of individual dipoles. Hemispheric homology is just the structure we expect as a compromise between difference and speed of interaction.
} 
this concept to entrainment in dyadic interactions (e.g. Abney et al. 2014, 2015; Marmelat and Delignières 2012). ${ }^{3}$

Goodfellow et al. (2014) exploit some aspects of hemisphericity in Generative Adversarial Networks (GANs). In a GAN, one network generates and the other discriminates; the generator attempts to increase the error rate of the discriminator by getting it to accept spurious synthesized examples as genuine data from the training set. Zhang, Isola and Efros' (2016) aptly named 'split-brain autoencoders' carry out unsupervised learning in which one channel must predict the contents of the other. The success of these machine-learning approaches in real-world computer vision tasks speaks to the utility of a general schema of interaction between two relatively encapsulated processors.

\section{Mirror Neurons, Predictive Processing and the Hemispheres}

We now look at a previously little-considered aspect of the mirror-neuron system (MNS) - its potential role in hemispheric communication, such that mirror neurons (at least for part of their history) mediate for one hemisphere the interpretation of visible and/or audible activities controlled by the other hemisphere. Thus, for instance, the RH initiates reaching for the teacup with the left hand, the eyes follow this activity (cf. Land and Hayhoe 2001) and the LH MNS conveys to the rest of the LH the effective gist of the behaviour of the RH.

Researchers have studied the laterality of the MNS with respect to sense of self (see Uddin et al. 2007), the origins of (left-lateralized) language (see Aziz-Zadeh et al. 2004; Rizzolatti and Arbib 1998), speech perception (e.g. Lotto et al. 2009), empathy and 'mind-reading' (e.g. Gallese and Goldman 1998; Iacoboni 2009), but very few studies have involved distinguishing stimuli and responses by hemisphere/ hemifield, with a primary research focus on the role of the hemispheres. One exception is a study by Aziz-Zadeh et al. (2006), who presented egocentrically positioned representations of a hand in front of human participants and recorded fMRI data as the participant observed, imitated, or executed actions in response to the hand, which might or might not move to the left or right to press a button. The authors were testing the hypothesis that the MNS would be left-lateralized, meaning it could be an evolutionary precursor of spoken language. The data showed significant differences in activity, specifically during imitation; activity in the inferior frontal and rostral inferior parietal cortex was stronger in the hemisphere ipsilateral to the visual stimulus and response hand. The authors saw these data as "paradoxical" (p. 2969) in terms of the typical contralateral projections.

Increased ipsilateral activity accompanying imitation is consonant with our proposal that the MNS is involved in interhemspheric coordination. However, comparable MNS data remain scant (although see Levy 1977, p. 91, as cited by Schechter

\footnotetext{
3 An associated philosophy-of-science conclusion regarding "explanatory pluralism" has been advanced by Abney et al. (2007), although it would appear to be at odds with the proposals regarding explanation implicit in the current paper.
} 
2015, p. 510): much MNS research has understandably been directed at non-human animals; hemisphericity has been largely ignored; the early development of the MNS, particularly relevant to the current claim, has received little attention; thresholding decisions may mask bilateral activity (and in the Aziz-Zadeh study, there is certainly bilateral activity); the very interpretation of activity in fMRI is ambiguous. Finally, as Aziz-Zadeh et al. note, there are complex departures from the standard picture of strict contralateral projection in adult non-MNS neural connectivity to and from the fingers and hands (Ehrsson et al. 2000); these departures favour weak precision grips (Ehrsson et al. 2001) suggesting their importance for bimanual manipulation.

The virtually exclusive concern with intersubjectivity in MNS research is not true of predictive coding, or predictive processing - 'PP', research (e.g. Leube et al. 2003) although the primary orientation is towards interacting with others (cf. Clark 2013; Friston 2005; Grush 2004; Rao and Ballard 1999; Lee and Mumford 2003). The idea that motor control, perception, imagery and beliefs might all be governed by internal-emulation/forward-modelling/efference-copying of the world/self/others has attracted wide research interest. These ideas have histories in control theory, in signal processing, in 'analysis-by-synthesis', and go at least as far back as Helmholtz's (1866/2004) ideas concerning unconscious inference; they now return with greater mathematical and neuroscientific content.

In summary, we note the potential utility of the MNS and of prediction error mechanisms for hemispheric coordination and the subsequent implications for the phylogenetic and ontogenetic development of cognition.

\section{Conclusions: Mutual Reflection of the Two Hemispheres}

We have begun to make the case that hemisphericity and the coordination it necessitates is the essential material precipitator of cognition. We now develop this basic hypothesis.

(1) The simplest relationship between the hemispheres is physical co-presence; indeed, Vesalius suggested a purely mechanical function for the corpus callosum, fastening together the right and left cerebra (Clarke and O'Malley 1968). Physical co-presence means largely shared internal and external environments and a plurality of means of coordinating the two hemispheres.

(2) One hemisphere's domain of activity is (pretty much) the individual's entire external-world together with (pretty much) the individual's entire somatic context, crucially including its partner hemisphere (cf. Schechter's definition of R and L, above). These shared domains are the material 'fulcrum' that allows the interaction of the two hemispheres, with complex, re-entrant loops of activity that involve the brain and the world outside the brain (cf. Clark 2009; Hurley 2002; Noë 2007; Schechter 2015; Thompson and Varela 2001).

(3) The most complex relationship between the hemispheres is where one hemisphere actively incorporates the other hemisphere's contextualized activity into its own repertoire. Mutual modelling of one hemisphere by the other necessar- 
ily ensues, such that each hemisphere operates on multiply-nested assumptions about both hemispheres.

(4) Nested coordinations are enhanced by hemispheric asymmetry: just as imperfect parallel mirrors generate idiosyncratic series of reflections, so the differently parameterized hemispheres with their lateralized specialisms have the capacity to create more idiosyncratic cognitive tools than would be possible in the symmetric case (which more resembles the other higher primates).

(5) Although such asymmetries are observable in gene-expression differences in human embryos in advance of any morphological difference (Sun et al. 2005), in evolution and particularly in individual development they are increasingly mediated by artefacts (including the hands-as-tools, tools and language) and social interaction.

(6) Although Sun et al.'s reported asymmetry also occurs in mice, it does so without a directional preference (i.e. it operates at the individual level, producing a 50:50 population), as noted by Fisher and Marcus (2006). We see the beginnings of a critical population-wide lateralization in brain structure in nonhuman primates (Cantalupo and Hopkins 2001; Gannon et al. 1998); such an asymmetry, as in handedness, is consonant with the brain-to-brain (or, more accurately, hemisphere-to-hemisphere) coordination involved in intersubjectivity and collaboration, as we saw above for face-to-face collaboration. This situation exemplifies the prioritizing of hemispheres over whole brains in the theorizing we present here.

(7) The human visual system has evolved so as to carry the hemispheric divide to its furthest outpost, the fovea. The vertical division and subsequent contralateral hemispheric projection of the visual field extends into our highest-fidelity, most detailed visual processing (see, e.g., Ellis and Brysbaert 2010; Shillcock et al. 2000). The retina, including the fovea, packages up the visual world to exploit hemisphericity.

(8) Hemispheric lateralization is an arms race. Hemispheric encapsulation, asymmetry and mutual modelling create a virtuous spiral of increasingly sophisticated outcomes of mutual modelling, which constitute 'tools of cognition'. These hemisphere-specific tools interact in human cognitive activity.

(9) This virtuous spiral to produce cognitive tools is a gamble. A further twist of the spiral (i.e. greater encapsulation, more lateralization) might achieve even deeper mutual modelling and more sophisticated tools to be coordinated between the two hemispheres, but it also risks disrupting even the current level of coordination. There is an inverse relation between brain lateralization and callosal connectivity in males (Aboitiz et al. 1992; Dorion et al. 2000; Witelson and Goldsmith 1991), suggesting that we should see in males a greater variability at both the upper and lower ends of the range of cognitive performance, which is exactly what we do see (Johnson et al. 2008). (See Shillcock et al. 2018, for the relevant cognitive modelling.)

(10) Cognitive scientists have conventionally prioritized 'mindreading'/intersubjectivity over metacognition/'intrasubjectivity', even deriving the latter from the former (e.g. Carruthers 2009). We argue that the rich, constant 24/7 connectivity between the hemispheres, grounded by perceptual duplication of 
their input, means that they are driven to develop sophisticated models of each other's activities, based on mechanisms such as the MNS and prediction-error/ emulation. The implications of this move for our understanding of cognition are profound.

We have traced an argument that the material requirement for the coordination of the two hemispheres forces the development of the wherewithal for higher cognition, phylogenetically and ontogenetically. The highest achievement in the development of cognition is arguably human consciousness. What are the implications of our analysis - relying on relative hemispheric autonomy-for the unity of consciousness, which has been a consistent concern of philosophers (Brook and Raymont 2017)? How is it that conscious experience is a single whole as opposed to an independent registering of different dimensions and entities? In some of the claimed subtypes of unity of consciousness, this issue equates to the binding problem (Hardcastle 1998; Revonsuo 1999).

Consider that there are aspects of cognitive processing that are specific to a particular hemisphere. The WADA test allows the clinician to render one or other hemisphere of a patient inactive by introducing a barbiturate into one of the internal carotid arteries (Wada 1949). Patients typically anecdotally report feeling no different from how they normally feel, but are aware of failures to comprehend language, to produce language, to remember test items, and to hold up an affected arm (Epilepsy Foundation, Community Forum 2018). They report no memory of the affected hemisphere 'coming back'. How can we understand this unified consciousness?

We see the general problem of the unity of consciousness as at least partly finessed by the (possibly multiply nested) mutual modelling of the two hemispheres; each hemisphere predicts aspects of the way that the other hemisphere interacts with the world. We presume that the seeming equipotential, indivisible nature of the WADA-test patients' conscious awareness reflects the unaffected hemisphere's modelling of the affected hemisphere.

We have argued above that the maximal 'bandwidth' of the interaction between the hemispheres makes their role a critical one, compared with the interaction between smaller brain regions in the same hemisphere, for instance. However, we do not restrict the role for predictive processing to interhemispheric interaction. Considering just vision, the pervasive recurrent connectivity in the visual areas of the brain (cf. Felleman and Van Essen 1991), both cortical and subcortical, provide ample opportunity for predictive processing between different visual channels of information.

To illustrate, Millidge and Shillcock (2018) show that when a ('deep learning') convolutional neural network (cf. Zhang et al. 2016) is trained to make mutual predictions between different visual channels, such as the red versus green information in a scene, the resulting error maps (i.e. the incorrectly predicted pixels) strongly resemble the 'visual salience' maps that psychologists have used to capture where a viewer will fixate in a scene (e.g. Itti et al. 1998). Millidge and Shillcock reinterpret visual salience as visual informativeness/unpredictability.

We generalize our current position regarding the hemispheres to say that predictive processing between any and all different channels of perceptuo-cognitive 
processing serves to 'parse' the world and to underwrite the unity of consciousness. Nevertheless, because of the bandwidth observation, the hemispheric divide remains paramount; we have idealized the two hemispheres, in some of our considerations, as 'two brains in one bodily and world context'. Another aspect of the bandwidth issue is the question of which hemisphere takes control in particular task circumstances. Levy and Trevarthen (1976) coin the term 'metacontrol' to describe the fact that the dominant hemisphere in a particular situation is not necessarily dictated by the respective processing strengths and abilities of the two hemispheres, but by "values, knowledge, expectations and intentions" (p. 311) intrinsic to the active subject.

Prioritizing the development of interhemispheric coordination in the understanding of cognition solves the problem of how a system can recursively organize itself in a spiral of open-ended engagement with a world very largely shared by the two hemispheres while at the same time being able to bootstrap itself into more and more complex behaviour by virtue of those same hemispheres observing each other. In this sense, the hemispherically-divided brain is a self-creating and self-organizing system coupled to the world (cf. Maturana and Varela 1980; Varela et al. 1974) not just by the direct predictions of the predictive processing approach, but also by the computationally powerful 'second-order' predictions of one hemisphere predictively modelling the other.

In summary, we have taken the totality of the cognizing individual and their world and we have abstracted away everything except the two hemispheres and the necessity that they be coordinated. This coordination presents unique opportunities for the emergence of human cognition. Theorizing about intersubjectivity and 'intrasubjectivity' in terms of hemispheres rather than whole brains is profoundly productive.

Open Access This article is distributed under the terms of the Creative Commons Attribution 4.0 International License (http://creativecommons.org/licenses/by/4.0/), which permits unrestricted use, distribution, and reproduction in any medium, provided you give appropriate credit to the original author(s) and the source, provide a link to the Creative Commons license, and indicate if changes were made.

\section{References}

Abney DH, Dale R, Yoshimi J, Kello CT, Tylén K, Fusaroli R (2007) Joint perceptual decision-making: a case study in explanatory pluralism. Front Psychol Theor Philos Psychol 5:330. https://doi. org/10.3389/fpsyg.2014.00330

Abney DH, Paxton A, Dale R, Kello CT (2014) Complexity matching in dyadic conversation. J Exp Psychol Gen. https://doi.org/10.1037/xge0000021

Abney DH, Kello CT, Warlaumont AS (2015) Production and convergence of multiscale clustering in speech. Ecol Psychol 27(3):222-235

Aboitiz F, Montiel J (2003) One hundred million years of interhemispheric communication: the history of the corpus callosum. Braz J Med Biol Res 36(4):409-420

Aboitiz F, Scheibel AB, Zaidel E (1992) Morphometry of the Sylvian fissure and the corpus callosum, with emphasis on sex differences. Brain 115(5):1521-1541

Allegrini P, Bologna M, Grigolini P, West BJ (2006). Response of complex systems to complex perturbations: the complexity matching effect. arXiv preprint arXiv:cond-mat/0612303

Amunts K, Jäncke L, Mohlberg H, Steinmetz H, Zilles K (2000) Interhemispheric asymmetry of the human motor cortex related to handedness and gender. Neuropsychologia 38(3):304-312 
Anninos PA, Cook ND (1988) Neural net simulation of the corpus callosum. Int J Neurosci 38(3-4):381-391

Aziz-Zadeh L, Iacoboni M, Zaidel E, Wilson S, Mazziotta J (2004) Left hemisphere motor facilitation in response to manual action sounds. Eur J Neurosci 19(9):2609-2612

Aziz-Zadeh L, Koski L, Zaidel E, Mazziotta J, Iacoboni M (2006) Lateralization of the human mirror neuron system. J Neurosci 26(11):2964-2970

Beeman M, Chiarello C (1998) Right hemisphere language comprehension: perspectives from cognitive neuroscience. Psychology Press, Hove

Beeman M, Friedman RB, Grafman E, Perez E (1994) Summation priming and coarse semantic coding in the right hemisphere. J Cogn Neurosci 6:26-45

Berlucchi G, Antonini A (1990) The role of the corpus callosum in the representation of the visual field in cortical areas. In: Trevarthen C (ed) Brain circuits and functions of the mind: essays in honor of Roger W. Sperry. Cambridge University Press, Cambridge, pp 129-137

Bogen J (1969) The other side of the brain II: an appositional mind. Bull Los Angel Neurol Soc $34: 135-162$

Brook A, Raymont P (2017) The unity of consciousness. In: Zalta EN (ed) The Stanford encyclopedia of philosophy (Summer 2017 Edition). https://plato.stanford.edu/entries/consciousness-unity/. Retrieved 23 Nov 2018

Cantalupo C, Hopkins WD (2001) Asymmetric Broca's area in great apes. Nature 414:505

Carruthers P (2009) How we know our own minds: the relationship between mindreading and metacognition. Behav Brain Sci 32(02):121-138

Cashmore L, Uomini N, Chapelain A (2008) The evolution of handedness in humans and great apes: a review and current issues. J Anthropol Sci 86:7-35

Cavanagh T, Berbesque JC, Wood B, Marlowe F (2015) Hadza handedness: lateralized behaviors in a contemporary hunter-gatherer population. Evol Hum Behav 37:202-209

Chalmers D (2008) Foreword to Clark, A. (2008) Supersizing the mind: embodiment, action, and cognitive extension. Oxford University Press, Oxford, pp ix-xvi

Chiarello C (1980) A house divided? Cognitive functioning with callosal agenesis. Brain Lang 11(1):128-158

Chiarello C, Maxfield L (1996) Varieties of interhemispheric inhibition, or how to keep a good hemisphere down. Brain Cogn 30(1):81-108

Clark A (2009) Spreading the joy? Why the machinery of consciousness is (probably) still in the head. Mind 118(472):963-993

Clark A (2013) Whatever next? Predictive brains, situated agents, and the future of cognitive science. Behav Brain Sci 36(03):181-204

Clarke E, O'Malley CD (1968) The human brain and spinal cord. University of California Press, Berkeley

Cook ND (1986) The brain code: mechanisms of information transfer and the role of the corpus callosum. Methuen, London

Davis L (1997) Cerebral hemispheres. Philos Stud 87:207-222

Davis SW, Cabeza R (2015) Cross-hemispheric collaboration and segregation associated with task difficulty as revealed by structural and functional connectivity. J Neurosci 35(21):8191-8200

Denenberg VH (1981) Hemispheric laterality in animals and the effects of early experience. Behav Brain Sci 4(01):1-21

Dorion AA, Chantôme M, Hasboun D, Zouaoui A, Marsault C, Capron C, Duyme M (2000) Hemispheric asymmetry and corpus callosum morphometry: a magnetic resonance imaging study. Neurosci Res 36(1):9-13

Ehrsson HH, Fagergren A, Jonsson T, Westling G, Johansson RS, Forssberg H (2000) Cortical activity in precision-versus power-grip tasks: an fMRI study. J Neurophysiol 83(1):528-536

Ehrsson HH, Fagergren A, Forssberg H (2001) Differential fronto-parietal activation depending on force used in a precision grip task: an fMRI study. J Neurophysiol 85(6):2613-2623

Eliasmith C (2008) Dynamics, control, and cognition. In: Aydede ME, Robbins PE (eds) Cambridge handbook of situated cognition. Cambridge University Press, New York

Ellis AW, Brysbaert M (2010) Split fovea theory and the role of the two cerebral hemispheres in reading: a review of the evidence. Neuropsychologia 48(2):353-365

Epilepsy Foundation, Community Forum (2018) Messages posted to https://www.epilepsy.com/connect/ forums/surgery-and-devices/wada-testing. Retrieved 23 Nov 2018

Fadiga L, Fogassi L, Pavesi G, Rizzolatti G (1995) Motor facilitation during action observation: a magnetic stimulation study. J Neurophysiol 73(6):2608-2611 
Felleman DJ, Van Essen DC (1991) Distributed hierarchical processing in the primate cerebral cortex. Cereb Cortex 1(1):1-47

Fisher SE, Marcus GF (2006) The eloquent ape: genes, brains and the evolution of language. Nat Rev Genet 7(1):9-20

Friston K (2005) A theory of cortical responses. Philos Trans R Soc Lond B Biol Sci 360(1456):815-836

Gallese V, Goldman A (1998) Mirror neurons and the simulation theory of mind-reading. Trends Cogn Sci 2(12):493-501

Gallese V, Fadiga L, Fogassi L, Rizzolatti G (1996) Action recognition in the premotor cortex. Brain 119(2):593-609

Gannon PJ, Holloway RL, Broadfield DC, Braun AR (1998) Asymmetry of chimpanzee planum temporale: humanlike pattern of Wernicke's brain language area homolog. Science 279(5348):220-222

Gazzaniga M (1970) The bisected brain. Appleton-Century-Crofts, New York

Gazzaniga MS (2000) Cerebral specialization and interhemispheric communication. Brain 123(7):1293-1326

Gazzaniga MS (2002) Brain and conscious experience. In: Cacioppo JP (ed) Foundations in social neuroscience. MIT Press, Cambridge

Gazzaniga MS (2005) Forty-five years of split-brain research and still going strong. Nat Rev Neurosci 6(8):653-659

Gazzaniga MS, Sperry RW (1967) Language after section of the cerebral commissures. Brain 90(1):131-148

Gazzaniga MS, Bogen JE, Sperry RW (1962) Some functional effects of sectioning the cerebral commissures in man. Proc Natl Acad Sci 48(10):1765-1769

Gazzaniga MS, Bogen JE, Sperry RW (1963) Laterality effects in somesthesis following cerebral commissurotomy in man. Neuropsychologia 1(3):209-215

Gazzaniga MS, Bogen JE, Sperry RW (1965) Observations on visual perception after disconnexion of the cerebral hemispheres in man. Brain 88(2):221-236

Glickstein M (2009) Paradoxical inter-hemispheric transfer after section of the cerebral commissures. Exp Brain Res 192:425-429

Goodfellow I, Pouget-Abadie J, Mirza M, Xu B, Warde-Farley D, Ozair S, Courville A, Bengio Y (2014) Generative adversarial nets. Adv Neural Inf Process Syst 27:2672-2680

Grush R (2004) The emulation theory of representation: motor control, imagery, and perception. Behav Brain Sci 27:377-442

Hardcastle VG (1998) The binding problem. In: Bechtel W, Graham G (eds) A companion to cognitive science. Blackwell Publishers, Oxford, pp 255-265

Helmholtz HV (1866/2004) Treatise on physiological optics. Dover Press, Mineola

Hertz-Pannier L, Chiron C, Jambaqué I, Renaux-Kieffer V, Van de Moortele PF, Delalande O, Le Bihan D (2002) Late plasticity for language in a child's non-dominant hemisphere. Brain 125(2):361-372

Hobert O, Johnston RJ, Chang S (2002) Left-right asymmetry in the nervous system: the Caenorhabditis elegans model. Nat Rev Neurosci 3(8):629-640

Hopkins WD, Rilling JK (2000) A comparative MRI study of the relationship between neuroanatomical asymmetry and interhemispheric connectivity in primates: implication for the evolution of functional asymmetries. Behav Neurosci 114(4):739-748

Hurley SL (2002) Consciousness in action. Harvard University Press, Cambridge

Iacoboni M (2009) Imitation, empathy, and mirror neurons. Annu Rev Psychol 60:653-670

Itti L, Koch C, Niebur E (1998) A model of saliency-based visual attention for rapid scene analysis. IEEE Trans Pattern Anal Mach Intell 20(11):1254-1259

Johnson W, Carothers A, Deary IJ (2008) Sex differences in variability in general intelligence: a new look at the old question. Perspect Psychol Sci 3(6):518-531

Kingstone A, Gazzaniga MS (1995) Subcortical transfer of higher order information: more illusory than real? Neuropsychology 9(3):321-328

Kolb B, Whishaw IQ (1989) Plasticity in the neocortex: mechanisms underlying recovery from early brain damage. Prog Neurobiol 32(4):235-276

Land MF, Hayhoe M (2001) In what ways do eye movements contribute to everyday activities? Vis Res 41(25):3559-3565

Lee TS, Mumford D (2003) Hierarchical Bayesian inference in the visual cortex. J Opt Soc Am A 20(7):1434-1448

Leube DT, Knoblich G, Erb M, Grodd W, Bartels M, Kircher TT (2003) The neural correlates of perceiving one's own movements. Neuroimage 20(4):2084-2090 
Levy J (1977) Manifestations and implications of shifting hemi-inattention in commissurotomy patients. Adv Neurol 18:83-92

Levy J, Trevarthen C (1976) Metacontrol of hemispheric function in human split-brain patients. J Exp Psychol Hum Percept Perform 2(3):299-312

Loeser JD, Alvord EC Jr (1968) Clinicopathological correlations in agenesis of the corpus callosum. Neurology 18(8):745-756

Lotto AJ, Hickok GS, Holt LL (2009) Reflections on mirror neurons and speech perception. Trends Cogn Sci 13(3):110-114

MacNeilage PF, Rogers LJ, Vallortigara G (2009) Origins of the left and right brain. Sci Am 301(1):60-67

Marcano-Reik AJ, Blumberg MS (2008) The corpus callosum modulates spindle-burst activity within homotopic regions of somatosensory cortex in newborn rats. Eur J Neurosci 28(8):1457-1466

Marks C (1981) Commissurotomy, consciousness and unity of mind. MIT Press, Cambridge

Marmelat V, Delignières D (2012) Strong anticipation: complexity matching in interpersonal coordination. Exp Brain Res 222(1-2):137-148

Maturana HR, Varela FJ (1980) Problems in the neurophysiology of cognition. In: Autopoiesis and Cognition. Springer, Dordrecht, pp 41-47

Mevorach C, Humphreys GW, Shalev L (2005) Attending to local form while ignoring global aspects depends on handedness: evidence from TMS. Nat Neurosci 8(3):276-277

Middleton FA, Strick PL (2000) Basal ganglia and cerebellar loops: motor and cognitive circuits. Brain Res Rev 31(2):236-250

Millidge B, Shillcock R (2018) A predictive processing account of bottom-up visual saliency using crosspredicting auto-encoders. arXiv database. Retrieved 31 Aug 2018

Min BK, Marzelli MJ, Yoo SS (2010) Neuroimaging-based approaches in the brain- computer interface. Trends Biotechnol 28(11):552-560

Monaghan P, Pollmann S (2003) Division of labor between the hemispheres for complex but not simple tasks: an implemented connectionist model. J Exp Psychol Gen 132(3):379-399

Monaghan P, Shillcock R, McDonald S (2004) Hemispheric asymmetries in the split-fovea model of semantic processing. Brain Lang 88(3):339-354

Noë A (2007) Magic realism and the limits of intelligibility: what makes us conscious? Philos Perspect 21(1):457-474

Pais-Vieira M, Lebedev M, Kunicki C, Wang J, Nicolelis MAL (2013) A brain-to-brain interface for realtime sharing of sensorimotor information. Sci Rep. https://doi.org/10.1038/srep01319

Pashler H, Luck SJ, Hillyard SA, Mangun GR, O'Brien S, Gazzaniga MS (1994) Sequential operation of disconnected cerebral hemispheres in split-brain patients. Neuroreport 5(17):2381-2384

Pulsifer MB, Brandt J, Salorio CF, Vining EP, Carson BS, Freeman JM (2004) The cognitive outcome of hemispherectomy in 71 children. Epilepsia 45(3):243-254

Quaresmini C, Forrester GS, Spiezio C, Vallortigara G (2014) Social environment elicits lateralized behaviors in gorillas (gorilla gorilla gorilla) and chimpanzees (pan troglodytes). J Comp Psychol. https://doi.org/10.1037/a0036355

Rao RP, Ballard DH (1999) Predictive coding in the visual cortex: a functional interpretation of some extra-classical receptive-field effects. Nat Neurosci 2(1):79-87

Rao RP, Stocco A, Bryan M, Sarma D, Youngquist TM, Wu J, Prat CS (2014) A direct brain-to-brain interface in humans. PLoS ONE 9(11):e111332. https://doi.org/10.1371/journal.pone.0111332

Reggia JA, Goodall S, Shkuro Y (1998) Computational studies of lateralization of phoneme sequence generation. Neural Comput 10(5):1277-1297

Reggia JA, Goodall SM, Shkuro Y, Glezer M (2013) The callosal dilemma: explaining diaschisis in the context of hemispheric rivalry via a neural network model. Neurol Res 23:465-471

Revonsuo A (1999) Binding and the phenomenal unity of consciousness. Conscious Cogn 8(2):173-185

Rilling JK, Insel TR (1999) Differential expansion of neural projection systems in primate brain evolution. Neuroreport 10(7):1453-1459

Ringo JL, Doty RW, Demeter S, Simard PY (1994) Time is of the essence: a conjecture that hemispheric specialization arises from interhemispheric conduction delay. Cereb Cortex 4(4):331-343

Rizzolatti G, Arbib MA (1998) Language within our grasp. Trends Neurosci 21(5):188-194

Sauerwein HC, Nolin P, Lassonde M (1994) Cognitive functioning in callosal agenesis. In: Lassonde M, Jeeves MA (eds) Callosal agenesis: a natural split brain?. Plenum, New York, pp 221-233

Savaki H, Kennedy L, Sokolo L, Mishkin M (1996) Visually guided movement with the forelimb contralateral to a 'blind' hemisphere: contribution of the cerebellum. Neuroscience 75:143-159 
Schechter E (2013) Two unities of consciousness. Eur J Philos 21(2):197-218

Schechter E (2015) The subject in neuropsychology: individuating minds in the split-brain case. Mind Lang 30(5):501-525

Schlaug G, Jäncke L, Huang Y, Staiger JF, Steinmetz H (1995) Increased corpus callosum size in musicians. Neuropsychologia 33(8):1047-1055

Shillcock RC, Monaghan P (2001) Connectionist modelling of surface dyslexia based on foveal splitting: impaired pronunciation after only two half pints. In: Proceedings of the 23rd annual conference of the Cognitive Science Society

Shillcock R, Ellison TM, Monaghan P (2000) Eye-fixation behaviour, lexical storage and visual word recognition in a split processing model. Psychol Rev 107:824-851

Shillcock R, Bolenz F, Basak S, Morgan A, Fincham OED (2018) Understanding greater male variability in general intelligence: the role of hemispheric independence and lateralization. arXiv database. Retrieved 31 Aug 2018

Shkuro Y, Glezer M, Reggia JA (2000) Interhemispheric effects of simulated lesions in a neural model of single-word reading. Brain Lang 72(3):343-374

Sperry RW (1964) Problems outstanding in the evolution of brain function. James Arthur lecture on the evolution of the human brain, no. 33. American Museum of Natural History, New York

Sperry R (1965) Mind, brain and humanist values. In: Platt J (ed) New views of the nature of man. University of Chicago Press, Chicago

Sperry R, Gazzaniga M, Bogen J (1969) Interhemispheric relationships: the neo-cortical commissures; syndromes of hemisphere disconnection. In: Vinken P, Bruyn G (eds) Handbook of clinical neurology, vol 4. North-Holland Publishing, Amsterdam

Sun T, Patoine C, Abu-Khalil A, Visvader J, Sum E, Cherry TJ, Orkin SH, Geschwind DH, Walsh CA (2005) Early asymmetry of gene transcription in embryonic human left and right cerebral cortex. Science 308(5729):1794-1798. https://doi.org/10.1126/science.1110324

Thompson E, Varela F (2001) Radical embodiment: neural dynamics and consciousness. Trends Cogn Sci 5:418-425

Tong F (2003) Primary visual cortex and visual awareness. Nat Rev Neurosci 4(3):219-229

Trevarthen C (1990) Integrative functions of the cerebral commissures. Handb Neuropsychol 4:49-83

Tye M (2003) Consciousness and persons: unity and identity. MIT Press, Cambridge

Uddin LQ, Iacoboni M, Lange C, Keenan JP (2007) The self and social cognition: the role of cortical midline structures and mirror neurons. Trends Cogn Sci 11(4):153-157

Uddin L, Mooshagian E, Zaidel E, Scheres A, Margulies D, Clare Kelly A, Shehzad Z, Adelstein J, Castellanos F, Biswal B, Milham M (2008) Residual functional connectivity in the split-brain revealed with resting-state functional MRI. Neuroreport 19:703-709

Varela FG, Maturana HR, Uribe R (1974) Autopoiesis: the organization of living systems, its characterization and a model. Biosystems 5(4):187-196

Wada J (1949) A new method of determining the side of cerebral speech dominance: a preliminary report on the intracarotid injection of sodium amytal in man. Igaku to Seibutsugaku 14:221-222

West BJ, Geneston EL, Grigolini P (2008) Maximizing information exchange between complex networks. Phys Rep 468:1-99. https://doi.org/10.1016/j.physrep.2008.06.003

Whitteridge D (1965) Area 18 and the vertical meridian of the visual field. In: Ettlinger EG (ed) Functions of the corpus callosum. Churchill, London

Witelson SF, Goldsmith CH (1991) The relationship of hand preference to anatomy of the corpus callosum in men. Brain Res 545(1):175-182

Witelson SF, Pallie W (1973) Left hemisphere specialization for language in the newborn. Neuroanatomical evidence of asymmetry. Brain 96(3):641-646

Yoo SS, Kim H, Filandrianos E, Taghados SJ, Park S (2013) Non-invasive brain-to-brain interface (BBI): establishing functional links between two brains. PLoS ONE 8(4):e60410

Zhang R, Isola P, Efros AA (2016) Split-brain autoencoders: unsupervised learning by cross-channel prediction. arXiv preprint arXiv:1611.09842 